\title{
Análisis por elementos finitos de la deformación aplicada a la aleación de aluminio 6061-T6 mediante presión en canal angular de sección constante
}

\author{
Oscar Fabián Higuera-Cobos ${ }^{1}$ \\ Luis Carlos Flórez-García² \\ Cristian Antonio Pedraza-Yepes ${ }^{3}$
}

Fecha de recepción: 29 de octubre de 2018

Fecha de aprobación: 12 de diciembre de 2018

\section{Resumen}

El proceso de deformación plástica severa "presión en canal angular de sección constante (ECAP/ECAE por sus siglas en inglés)", se simuló bajo la teoría de elementos finitos utilizando el software ANSYS, aproximando respuestas elastoplásticas que se puedan presentar en una probeta de aleación de aluminio 6061-T6. Se estudiaron las variaciones de los parámetros mecánicos de la matriz ECAP, tales como su geometría (ángulo externo $(\Psi)$ y ángulo interno $(\Phi)$ ) y las condiciones de fricción del proceso. Se analizó la influencia de cada uno de estos parámetros en el comportamiento del material. Los resultados obtenidos fueron comparados con las predicciones teóricas que se han dispuesto a lo largo del estudio del ECAE/ECAP por parte de diferentes investigadores.

Palabras clave: aleación de aluminio; deformación; ECAE; programa de ingeniería; SPD.

\section{Finite elements analysis of the deformation applied to the 6061-T6 aluminum alloy by equal channel angular pressing}

\begin{abstract}
The severe plastic deformation process Equal Channel Angular Pressing (ECAP/ECAE), was simulated under the theory of finite elements using the software ANSYS, in search of different elastoplastic responses that can be presented to a sample of 6061-T6 aluminum alloy. Variations of the mechanical parameters of the ECAP matrix, such as its geometry (external angle $(\Psi)$ and internal angle $(\Phi))$ and the friction conditions of the process were studied. The influence of each of these parameters on the behavior of the material was analyzed. The results obtained were compared with the theoretical predictions that have been arranged throughout the ECAE / ECAP study by different researchers.
\end{abstract}

Keywords: aluminum alloy; ECAE; engineering software; SPD; strain.

\footnotetext{
${ }^{1}$ Ph. D. Universidad del Atlántico (Barranquilla-Atlántico, Colombia). oscarhiguera@mail.uniatlantico.edu.co. ORCID: 0000-0002-4836-5215.

${ }^{2}$ M. Sc. Universidad Tecnológica de Pereira (Pereira-Risaralda, Colombia). louis@utp.edu.co. ORCID: 0000-0002-58878006 .

${ }^{3}$ M. Sc. Universidad del Atlántico (Barranquilla-Atlántico, Colombia). cristianpedraza@mail.uniatlantico.edu.co. ORCID: 0000-0002-5951-7835.
}

Revista Facultad de Ingeniería (Rev. Fac. Ing.) Vol. 28 (50), pp. 7-18. Enero-Marzo, 2019. Tunja-Boyacá, Colombia. L-ISSN: 0121-1129, e-ISSN: 2357-5328, DOI: 
Análisis por elementos finitos de la deformación aplicada a la aleación de aluminio 6061-T6 mediante presión en canal angular de sección constante

\section{Análise por elementos finitos da deformação aplicada à liga de alumínio 6061-T6 mediante pressão em canal angular de seção constante}

\section{Resumo}

O processo de deformação plástica severa "pressão em canal angular de seção constante (ECAP/ECAE por suas siglas em inglês)", simulou-se sob a teoria de elementos finitos utilizando o software ANSYS, aproximando respostas elastoplásticas que se apresentem em uma proveta de liga de alumínio 6061-T6. Estudaram-se as variações dos parâmetros mecânicos da matriz ECAP, tais como sua geometria (ângulo externo $(\Psi)$ e ângulo interno $(\Phi)$ ) e as condições de fricção do processo. Analisou-se a influência de cada um destes parâmetros no comportamento do material. Os resultados obtidos foram comparados com as predições teóricas que tenham sido dispostas ao longo do estudo do ECAE/ECAP por parte de diferentes pesquisadores.

Palavras chave: deformação; ECAE; liga de alumínio; programa de engenharia; SPD.

\section{Para citar este artículo:}

O. F. Higuera-Cobos, L. C. Flórez-García, and C. A. Pedraza-Yepes, "Análisis por elementos finitos de la deformación aplicada a la aleación de aluminio 6061-T6 mediante presión en canal angular de sección constante," Revista Facultad de Ingeniería, vol. 28 (50), pp. 7-18, Ene. 2019. DOI: https://doi.org/10.19053/01211129.v28.n50.2019.8781.

Esta obra está bajo licencia internacional Creative Commons Reconocimiento 4.0

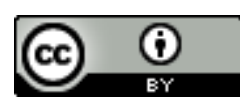




\section{Introducción}

Los materiales nanoestructurados son definidos como sólidos que cuentan con características microestructurales en el rango de 1-100 nm al menos en una dirección [1]. Los materiales nanoestructurados pueden ser sintetizados, ya sea, mediante la consolidación de pequeñas agrupaciones o por fragmentación microestructural del material másico policristalino en unidades cristalinas con dimensiones nanométricas. Estos procesos han sido clasificadas como "bottom up" y "top down", respectivamente. En el proceso "bottom up" la nanoestructura se ordena átomo por átomo o capa por capa. Por ejemplo, en esta categoría se incluyen técnicas como condensación en gas inerte, electrodeposición y deposición química y física. En el proceso "top down" se inicia con un material másico y se fragmenta la microestructura en una nanoestructura [2].

Las técnicas más destacadas incluyen el uso de deformación plástica severa, en la cual, los materiales son sometidos a una deformación muy grande sin la introducción de cambios significativos en las dimensiones de la sección transversal de las piezas. Varios métodos de procesamiento por deformación plástica severa (SPD) están disponibles actualmente, incluyendo presión en canal angular de sección constante (ECAP) [3,4], torsión alta presión (HPT) [5,6], unión por laminado acumulativo (ARB) [7,8], corrugado y enderezamiento repetitivo (RCS). Algunos intentos se han hecho para combinar algunos de estos procedimientos, tales como ECAP y laminado en frio, ARB y FSP o ECAP y HPT [9].

De las técnicas SPD anteriormente mencionadas, el procesamiento por ECAP tiene por lo menos dos ventajas que favorecen su adopción a nivel industrial. Primero, puede ser escalada para producir muestras másicas relativamente grandes. Segundo, puede ser incorporada en trenes de laminación convencional para procesamiento continuo. El proceso ECAP consiste en presionar una pieza metálica a través de una matriz metálica en donde dos canales se interceptan en un ángulo interno $(\Phi)$, comprendido entre $60^{\circ}$ y $160^{\circ}$ y en un ángulo externo $(\Psi)$ que puede estar comprendido entre $0^{\circ} \leq \psi \leq(\pi-\Phi)^{\circ}$ resultando una gran deformación por pase sobre las muestras metálicas, la cual puede ser estimada mediante aproximaciones analíticas basadas en las configuraciones geométricas de la matriz, como se muestra en la Figura 1.

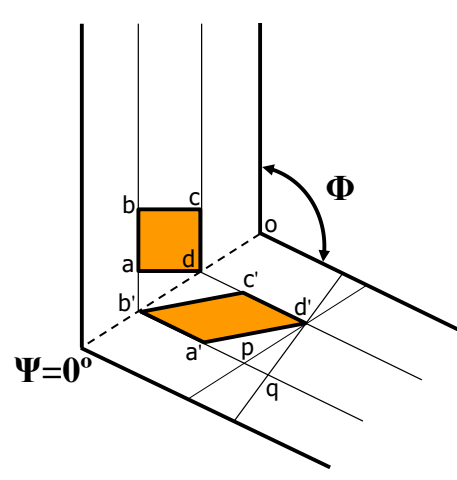

(a)

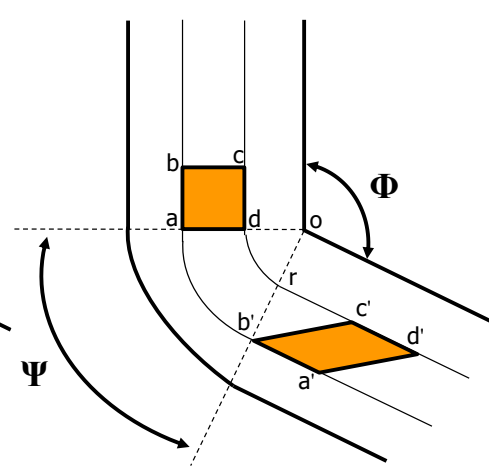

(b)

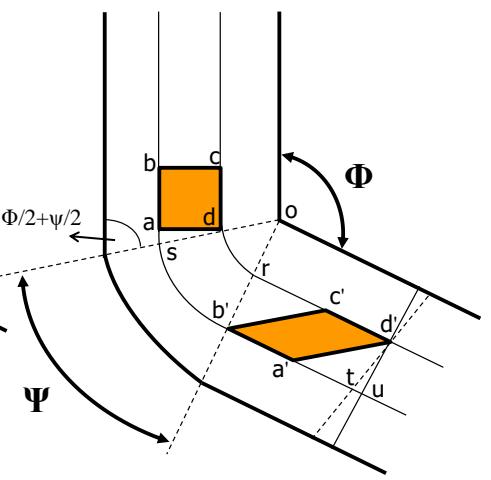

(c)

Fig. 1. Configuraciones de la matriz ECAP. (a) $\psi=0^{\circ}$, (b) $\psi=(\pi-\Phi)^{\circ}$, (c) $0^{\circ}<\psi<(\pi-\Phi)^{\circ}$ [3]. 
La Figura 1 plantea tres condiciones diferentes para el cálculo de la deformación de von Mises en función de la configuración de la matriz ECAP. Para la simulación de cada condición, se asumió que las probetas están completamente lubricadas, por lo tanto, los efectos de fricción fueron despreciados. Para el análisis se utilizó un elemento cuadrado situado en la entrada del canal, etiquetado como "abcd", que pasa a través de un plano de corte teórico y se distorsiona en el paralelogramo etiquetado como " $a$ ' $b$ 'c' $d$ ".

Caso 1: El cual corresponde a una situación límite en donde $\Psi=0^{\circ}$ (Figura 1a), la deformación cortante equivale $a \gamma=a^{\prime} q / q^{\prime} d^{\prime}$, en donde $\mathrm{qd}^{\prime}=a d$ y $a b^{\prime}=d c^{\prime}=a^{\prime} p=$ $\mathrm{pq}=\operatorname{adcot} \frac{\Phi}{2}$ y $\mathrm{a}^{\prime} \mathrm{q}=2 \operatorname{adcot} \frac{\Phi}{2}$, por lo tanto, " $\gamma$ " fue calculada por la siguiente relación [3]:

$$
\gamma=2 \cot \frac{\Phi}{2}
$$

Caso 2: El cual corresponde a la situación en donde $\Psi=(\pi-\Phi)^{\circ}$ (Figura 1b), la deformación cortante equivale a $\gamma=\mathrm{rc}^{\prime} / \mathrm{rb}^{\prime}$, en donde $r b^{\prime}=\mathrm{da}=(\mathrm{oa}-\mathrm{od}), \mathrm{ab}^{\prime}=\mathrm{dc} \mathrm{c}^{\prime}=$ oa $\Psi=\left(r^{\prime}+o d \Psi\right)$ y rc'=(oa-od $) \Psi$. Por lo tanto, " $\gamma$ " cumple la siguiente relación [3]:

$$
\gamma=\Psi
$$

Caso 3: El cual corresponde a la situación intermedia en donde $0^{\circ}<\psi<(\pi-\Phi)^{\circ}$ (Figura1c), la deformación cortante equivale a $\gamma=a^{\prime} u / d^{\prime} u$, en donde $d^{\prime} u=a d y$ $a^{\prime} u$ puede ser obtenida por las relaciones $a^{\prime} u=\left(a^{\prime} t+t u\right)=\left(r c^{\prime}+a s\right)$, y como as $=\operatorname{adcot}\left(\frac{\Phi}{2}+\frac{\psi}{2}\right), a^{\prime}=d_{c}^{\prime}=(\operatorname{as}+o s \Psi)=\left(r^{\prime}+o d \Psi\right),(o s-o d)=\operatorname{adcsc}\left(\frac{\Phi}{2}+\frac{\psi}{2}\right)$ y a'u $=$ $2 \operatorname{adcot}\left(\frac{\Phi}{2}+\frac{\psi}{2}\right)+a d \Psi \csc \left(\frac{\Phi}{2}+\frac{\psi}{2}\right)$. Por lo tanto, " $\gamma$ " cumple la siguiente relación [3]:

$$
\gamma=2 \cot \cdot\left(\frac{\Phi}{2}+\frac{\Psi}{2}\right)+\Psi \csc \left(\frac{\Phi}{2}+\frac{\Psi}{2}\right)
$$

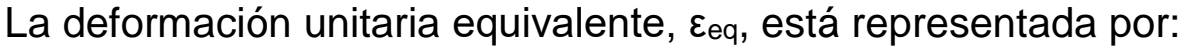

$$
\varepsilon_{\mathrm{eq}}=\left[\frac{2\left\{\varepsilon_{\mathrm{x}}^{2}+\varepsilon_{\mathrm{y}}^{2}+\varepsilon_{\mathrm{z}}^{2}+\frac{\gamma_{\mathrm{xy}}^{2}+\gamma_{\mathrm{yz}}^{2}+\gamma_{\mathrm{zx}}^{2}}{2}\right\}}{3}\right]^{1 / 2}
$$

Por lo tanto, la deformación $\varepsilon$, después de 1 pase es:

$$
\varepsilon=\left[\frac{2 \cot \left(\frac{\Phi}{2}+\frac{\Psi}{2}\right)+\Psi \csc \left(\frac{\Phi}{2}+\frac{\Psi}{2}\right)}{\sqrt{3}}\right]
$$

Y la deformación equivalente después de $\mathrm{N}$ pasadas por la matriz de ECAP, $\varepsilon_{N}$ está representada por la relación desarrollada por Iwahashi et al. [10]: 


$$
\varepsilon_{N}=\frac{N}{\sqrt{3}}\left[2 \cot \cdot\left(\frac{\Phi}{2}+\frac{\Psi}{2}\right)+\Psi \csc \cdot\left(\frac{\Phi}{2}+\frac{\Psi}{2}\right)\right]
$$

El objetivo de este trabajo es analizar el efecto de los parámetros de diseño de la matriz ECAP, tales como, ángulo interno $\left(\Phi=90^{\circ}, 120^{\circ}\right.$ y $\left.140^{\circ}\right)$ con un ángulo externo fijo $\left(\Psi=20^{\circ}\right)$ y las condiciones de fricción $(\mu=0,0.02,0.05,0.1,0.2,0.3$ y 0.32 ) sobre la distribución de esfuerzos en una probeta de la aleación de aluminio 6061-T6 procesada por ECAP.

\section{Modelamiento y simulación}

Haciendo uso del software ANSYS y su módulo de estática estructural, se llevó a cabo el modelamiento y la posterior simulación del proceso ECAP, basado en análisis 2D. Las variaciones de parámetros tales como fricción, ángulo externo $(\Psi)$ y ángulo interno $(\Phi)$ se realizaron con el fin de estudiar las diferentes respuestas elastoplásticas del material. Para esto, se utilizó una probeta de aluminio con dimensiones $70 \times 14 \times 14 \mathrm{~mm}$. Sus propiedades se resumen en la Tabla 1.

La Figura 2 muestra el esquema general de la matriz simulada. Para la simulación se utilizó un mallado de 966 elementos cuadriláteros iniciales de 1 $\mathrm{mm}$ en la probeta. Debido a las altas condiciones de deformación que se presentan en la probeta durante el procesado por ECAP, la calidad de la malla disminuye y se hace necesaria la implementación de un remallado durante la simulación, de manera que permita la compilación de esta.

Tabla 1. Propiedades de la aleación de Aluminio 6061-T6 utilizado en la simulación [11].

\begin{tabular}{|l|l|}
\hline \multicolumn{1}{|c|}{ Propiedad } & \multicolumn{1}{c|}{ Valor } \\
\hline Densidad & $2770 \mathrm{~kg} / \mathrm{m}^{3}$ \\
\hline Módulo de Young & $7,1 \times 10^{10} \mathrm{~Pa}$ \\
\hline Relación de Poisson & 0,33 \\
\hline Módulo de compresibilidad & $6,9608 \times 10^{10} \mathrm{~Pa}$ \\
\hline Módulo cortante & $2,6692 \times 10^{10} \mathrm{~Pa}$ \\
\hline Límite elástico & $2,8 \times 10^{8} \mathrm{~Pa}$ \\
\hline Módulo tangente & $5 \times 10^{8} \mathrm{~Pa}$ \\
\hline \multicolumn{2}{|c|}{${ }^{*}$ Propiedades para un comportamiento bilineal. } \\
\hline
\end{tabular}

De acuerdo con la Figura $2 \mathrm{a}$, d representa el desplazamiento de los nodos superiores de la probeta de $70 \mathrm{~mm}$ en la dirección $\mathrm{Y}$. Los cuerpos laterales son la estructura del canal, los cuales se fijaron de manera que no sufran desplazamiento ni rotación. Con este modelo se realizaron seis simulaciones, cada una de ellas variando los coeficientes de fricción $(\mu): 0,0.02,0.05,0.1,0.2$, 0.3 y 0.32 para diferentes ángulos internos $\left(\Phi=90^{\circ}, 120^{\circ}\right.$ y $\left.140^{\circ}\right)$ y con un ángulo externo fijo $\left(\Psi=20^{\circ}\right)$. 


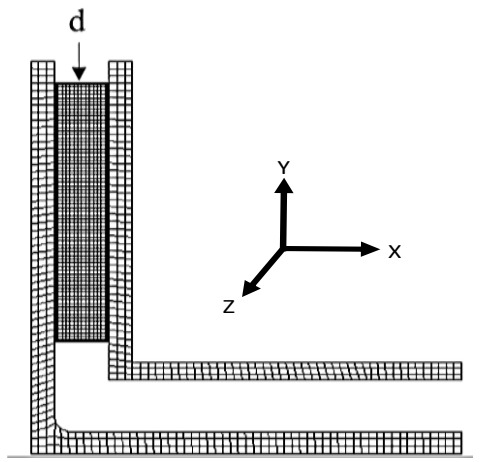

(a)

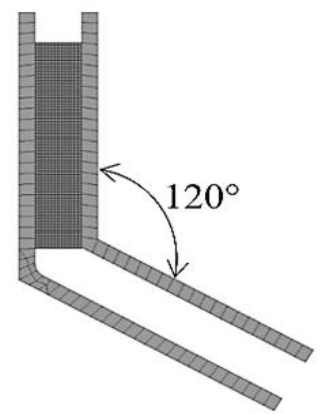

(b)

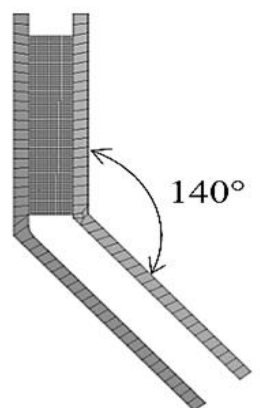

(c)

Fig. 2. Estado inicial de la simulación. (a) matriz con ángulo interno $\Phi=90^{\circ}$, (b) matriz con ángulo interno $\Phi=120^{\circ}$ y (c) matriz con ángulo interno $\Phi=140^{\circ}$.

\section{Resultados y análisis}

En la Figura 3 se muestran los resultados de la simulación al variar el coeficiente de fricción $(\mu)$ para una matriz ECAP con un ángulo interno de $\Phi=90^{\circ}$ y externo $\Psi=20^{\circ}$. De acuerdo con los planteamientos de Segal et al. [9] e Iwahashi et al. [10] descritos en la ecuación (6), la deformación impuesta al utilizar una matriz ECAP con esta configuración será de 1,05 por pase.
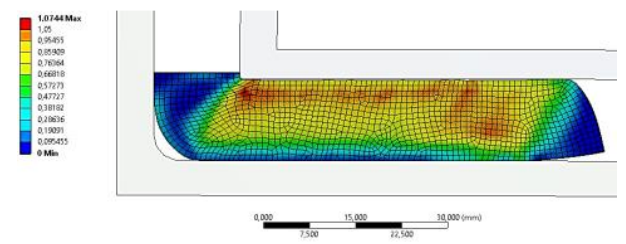

(a)
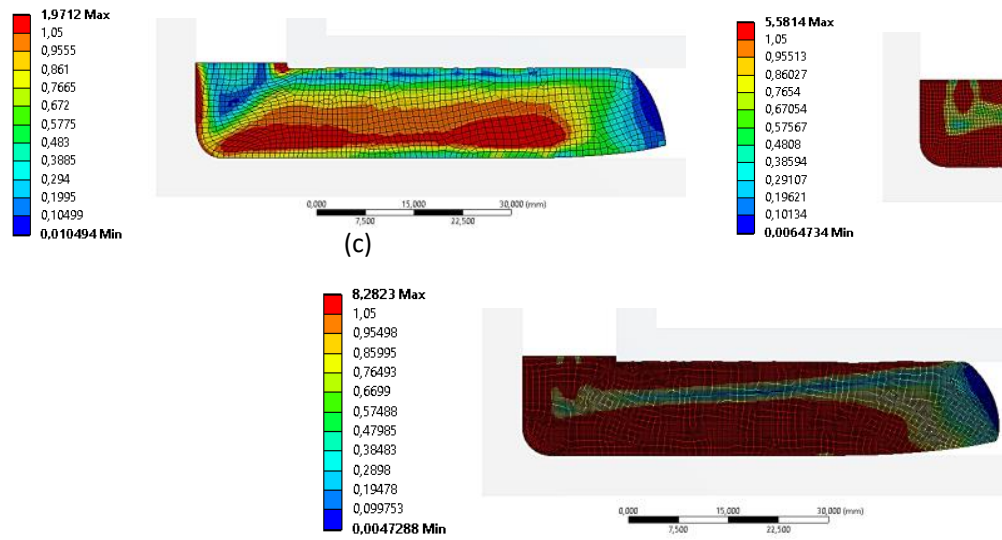

(e)

Fig. 3. Comportamiento plástico de la probeta de aluminio sometida a ECAP bajo diferentes condiciones de fricción. (a) $\mu=0$, (b) $\mu=0.1$, (c) $\mu=0.2$, (d) $\mu=0.3$ y (e) $\mu=0.32$.

En la Figura 3a se presentan los resultados de la simulación en condiciones ideales de deslizamiento $(\mu=0)$. Al ser un deslizamiento sin fricción, la probeta tiende a separar su contacto con la zona de redondeo de la matriz o la zona muerta del canal, dicho comportamiento se define como "Ilenado incompleto", lo cual impide que el cuerpo muestre la respuesta esperada por la teoría de Segal et al. [9], ya que éste no logra la deformación por cizalladura uniforme a lo largo 
de la probeta. Al incrementar el coeficiente de fricción a $\mu=0.1$ (Figura 3b) se presenta un incremento en la deformación máxima y en el llenado de la zona muerta, en comparación con el observado en la Figura 3a. En cuanto al coeficiente de fricción $\mu=0.2$ (Figura $3 c$ ), la deformación plástica equivalente máxima aumentó hasta $1,972 \mathrm{~mm} / \mathrm{mm}$ en la parte baja de la probeta (zona de redondeo de la matriz), mientras que, en la parte superior de la probeta, la deformación disminuyó, lo que podría deberse al llenado total de la zona muerta que minimiza el contacto al momento del giro en la arista superior. Un comportamiento similar fue reportado por Bowen et al. [12] al deformar aluminio de alta pureza mediante ECAE utilizando un matriz de $\phi=90^{\circ}$ y $\psi=0^{\circ}$, para dos condiciones distintas de fricción, una en la cual se asume deslizamiento ideal y otra donde se asume una fricción de 0,25. En dicho estudio se analizó únicamente el comportamiento visual del material durante la extrusión y cómo el mallado de éste se veía influenciado por el mismo. Las respuestas obtenidas muestran un comportamiento similar a los obtenidos en este trabajo para coeficientes de fricción de cero (Figura 3a y 4a) y 0,2 (Figura 3cy 4c). En cuanto a valores superiores del coeficiente de fricción $\mu=0.3$ (Figura 3d) y $\mu=0.32$ (Figura $3 e$ ) se obtuvieron valores muy superiores a los planteados teóricamente del orden de $5.5814 \mathrm{~mm} / \mathrm{mm}$ y $8.2823 \mathrm{~mm} / \mathrm{mm}$, respectivamente, localizados tanto en la parte superior e inferior de la probeta. Con el fin de verificar la homogeneidad de la deformación plástica se realizó una comparación entre la probeta completa (Figura 3) y una isocapa que muestra la región de la probeta que alcanzó por lo menos un 70\% de la deformación plástica teórica (Figura 4).

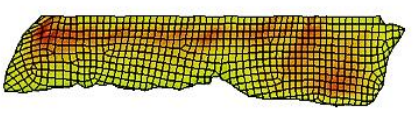

(a)

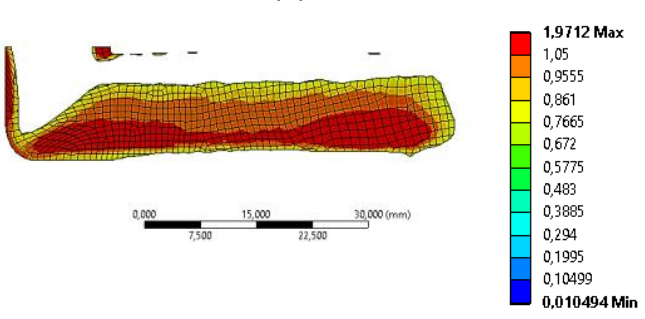

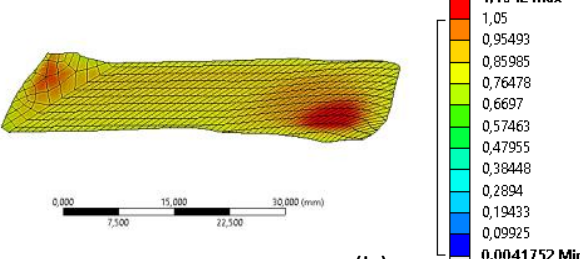

(b)
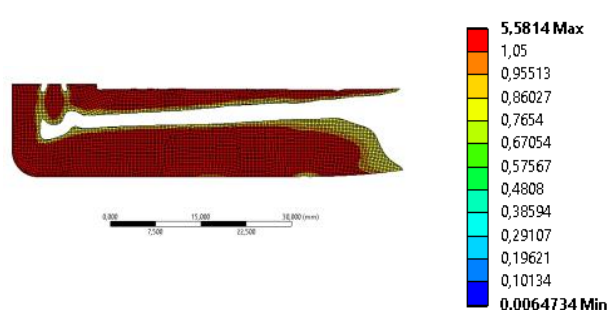

(c)

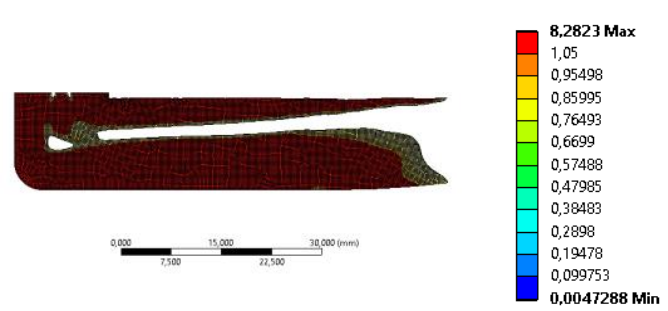

(d)

(e)

Fig. 4. Comparativa a isocapas de deformación 0,75 con un factor de fricción (a) $\mu=0$, (b) $\mu=0.1$, (c) $\mu=0.2$, (d) $\mu=0.3$ y (e) $\mu=0.32$. 
Las deformaciones plásticas logradas durante el proceso tuvieron una región parcialmente homogénea en el núcleo de la probeta, mientras en sus extremos fueron inferiores, lo cual supone que para un material procesado por ECAP, los extremos de la probeta deben ser descartados. Igualmente, la superficie de contacto de la muestra con el punto de giro de la matriz (borde superior) presentó deformaciones que, si bien fueron superiores a la de los extremos, siguen siendo muy bajas en comparación con las de su núcleo, reduciendo aún más la homogeneidad de las deformaciones logradas en el material. Las deformaciones obtenidas en la probeta comprendieron amplios rangos al aumentar la fricción. Para indicar una tendencia en el comportamiento se tomó el promedio de 10 muestras en el núcleo de la probeta en cada proceso para un coeficiente fricción dado. Dicho comportamiento se resume en la Figura 5 . En donde se observa una tendencia de incremento lineal hasta un coeficiente de fricción de 0,2. A partir de allí, las deformaciones del núcleo dejan de incrementar para concentrarse en la zona de contacto de la probeta. Sin embargo, con un coeficiente de fricción de 0,32 aparenta haber un gran salto en la deformación del núcleo lo que hace que la homogeneidad en las deformaciones plásticas de la probeta disminuya.

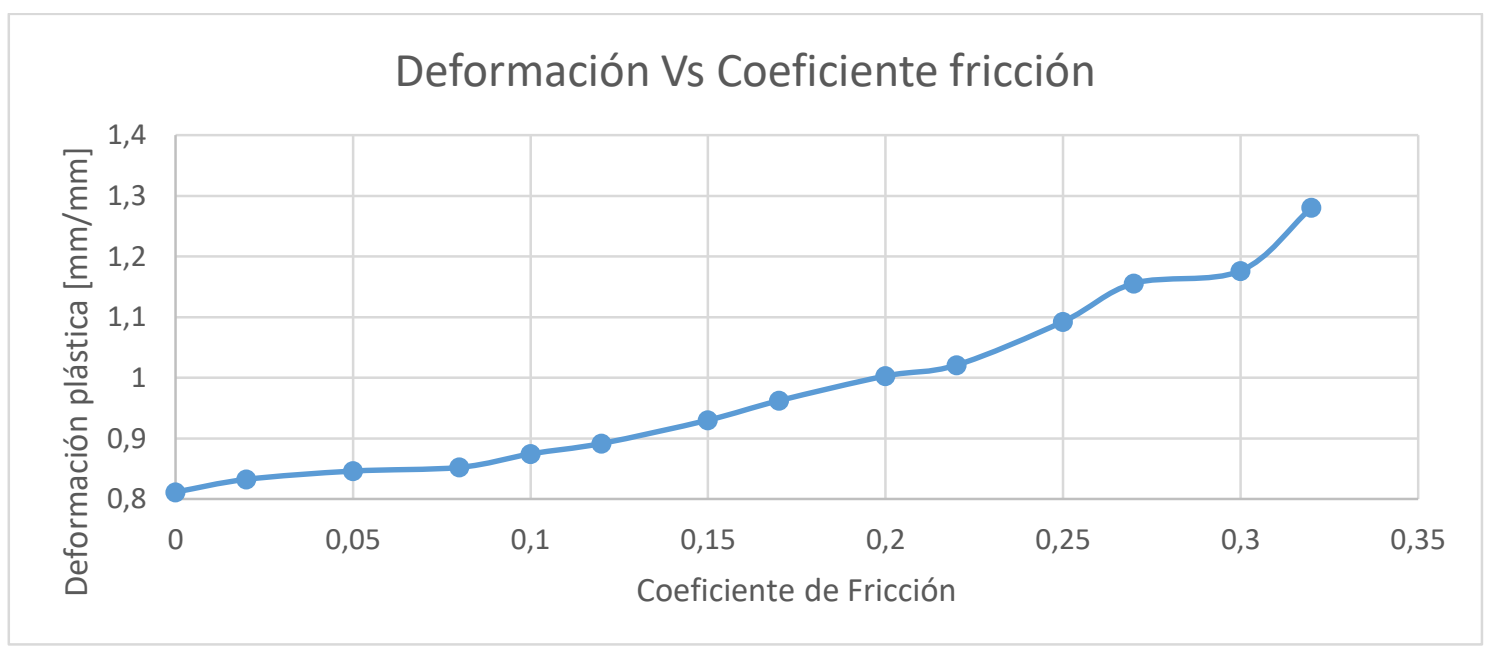

Fig. 5. Deformación plástica en función del coeficiente de fricción.

Al aumentar el ángulo interno a valores de $\Phi=120^{\circ}$ y $140^{\circ}$ con un ángulo externo de $\Psi=20^{\circ}$ se obtiene menores valores en la deformación aplicada a las muestras en comparación a la presentada en la Figura 3. Esto es coherente con lo planteado por Iwahashi et al. [10] en la ecuación (6). En donde para la matriz con una configuración $\Phi=120^{\circ}$ y $\Psi=20^{\circ}$ el resultado teórico es 0,635 por pase y para una matriz con una configuración $\Phi=140^{\circ}$ y $\Psi=20^{\circ}$ el resultado teórico es 0,408 por pase. Los resultados obtenidos se muestran en la Figura 6. La Figura 6a muestra un menor llenado de la matriz durante la deformación en comparación con el obtenido en la Figura 6b. Esto se debe principalmente a que el paso en la zona de giro es menos restrictivo para la probeta en la matriz con un ángulo interno mayor $\left(\Phi=140^{\circ}\right)$. En la matriz con ángulo interno de $\Phi=120^{\circ}$ la máxima deformación obtenida fue de $\varepsilon=0,72373$, la cual no está muy por encima de la teórica $(0,635)$. Aun así, mantiene la tendencia que tienen los bordes de la 
probeta a sufrir bajas deformaciones. Además, se puede observar una alta homogeneidad en la deformación en el núcleo, en donde más del $80 \%$ de la probeta obtuvo una deformación igual o superior al $70 \%$ de la deformación teórica.
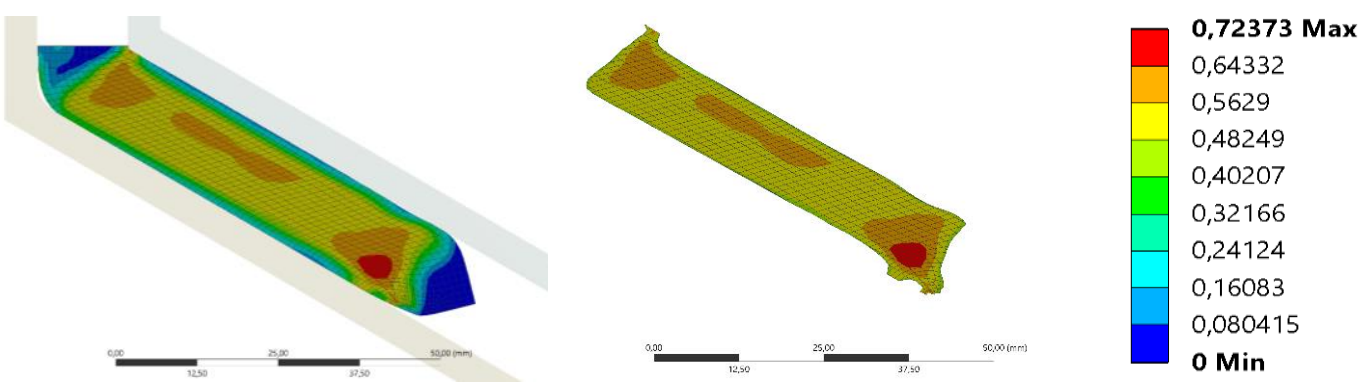

(a)
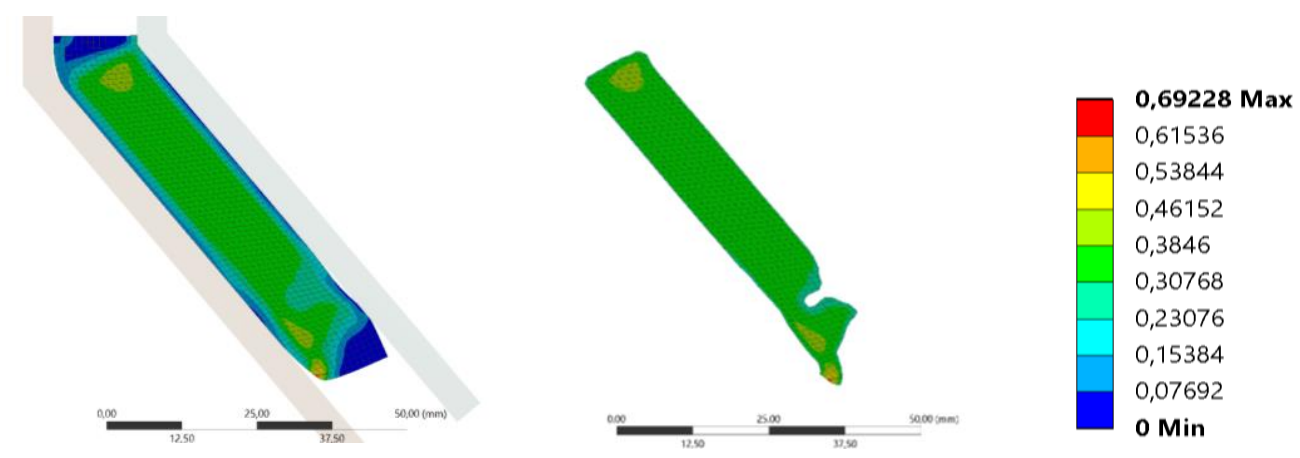

(b)

Fig. 6. Comportamiento plástico de la probeta de aluminio sometida a ECAP y Comparativa a isocapas de deformación 0,75 bajo condiciones ideales de fricción $(\mu=0)$. (a) $\Phi=120^{\circ}$ y (b) $140^{\circ}$ con un ángulo externo de $\Psi=20^{\circ}$.

En cuanto a la matriz con ángulo interno de $\Phi=140^{\circ}$ se observa como la uniformidad se mantiene en el núcleo de la probeta. La deformación máxima obtenida fue de $\varepsilon=0,69228$ concentrándose en el primer punto que hace contacto con el redondeo de la zona muerta, en este ángulo, ya no se generan deformaciones concentradas en el punto de contacto con la arista de $90^{\circ}$. Un comportamiento similar fue observado por Krishnaiah et al. [13] al procesar por ECAP cobre de alta pureza en una matriz con un ángulo interno de $120^{\circ} \mathrm{y}$ externo de $0^{\circ} y$ con un coeficiente de fricción $\mu=0.15$. Sin embargo, las deformaciones plásticas obtenidas en su estudio son superiores a las obtenidas en este trabajo. En conclusión, los resultados presentados en la Figura 6 confirman cómo al aumentar el ángulo y disminuir la restricción al flujo de la probeta, la deformación disminuye, pero su homogeneidad aumenta. Indicando un buen comportamiento y una buena similitud con las predicciones determinadas por Segal et al. [9] e Iwahashi et al. [10].

Las respuestas plásticas del material obtenidas con altas condiciones de fricción muestran comportamientos particulares donde se generan zonas de alta deformación plástica mientras en otras por el contrario disminuyen. Una 
justificación a dicho comportamiento se puede obtener a partir del comportamiento propuesto por Segal et al. [9], el cual se puede apreciar en la Figura $7 \mathrm{a}$, la cual describe claramente el comportamiento ideal de un cuerpo sometido a un proceso ECAE, en el cual no se presentan efectos de fricción. Allí se muestra cómo el ángulo abc del elemento previo al proceso de extrusión es de $90^{\circ}$ y se reduce una vez extruido. A partir de allí, se analiza la respuesta para un comportamiento donde se tienen en cuenta las condiciones de fricción mostrado en la Figura $7 \mathrm{~b}$, la cual ilustra el comportamiento de los elementos que presentan deformación por cizalladura generada por la fricción y el desplazamiento de la probeta.

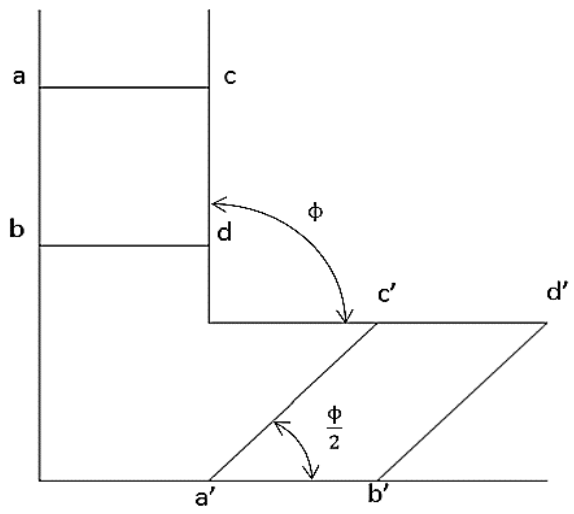

(a)

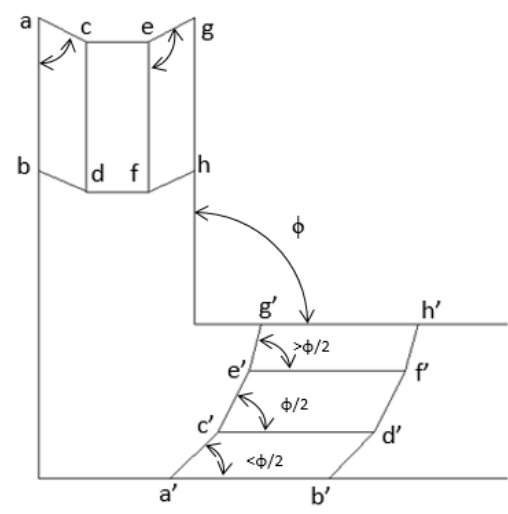

(b)

Fig. 7. Deformación por cizalladura generada por un proceso ECAE (a) sin fricción y (b) con fricción [9].

Los cuerpos 'abcd' y 'efgh' se encuentran bajo deformación por cizalladura previo al punto de giro generada por la fricción. En el esquema es posible ver como el ángulo efg antes del proceso es mayor a $90^{\circ}$ y una vez realizado el giro éste disminuye, acercándose al ángulo de $90^{\circ}$ donde la deformación por cizalladura disminuye. Por el contrario, el ángulo abc ya se encuentra comprendido en valores inferiores a $90^{\circ}$, por lo cual, el proceso de cizalladura del giro en la extrusión lo disminuye aún más, aumentando así su deformación plástica.

\section{Conclusiones}

Los resultados obtenidos en las simulaciones identificaron a la fricción como un factor que afecta directamente el comportamiento de la probeta en términos de deformación durante el proceso ECAP.

El coeficiente de fricción que generó la mejor relación entre uniformidad y magnitud de deformación fue 0,2. Razón por la cual fue el coeficiente utilizado para las condiciones de giro de $120^{\circ}$ y $140^{\circ}$.

La uniformidad de las deformaciones generadas en las probetas deformadas por ECAP en matrices con ángulos internos de $120^{\circ}$ y $140^{\circ}$ son superiores a las obtenidas para ángulos de $90^{\circ}$. Sin embargo, las magnitudes de sus 
deformaciones son inferiores, de acuerdo con lo determinado por Iwahashi [10] donde las deformaciones son acumulativas, y ellas aumentarán conforme se aumente el número de procesados, por lo cual al repetirse el procedimiento aumentará consecuentemente la deformación con una alta uniformidad.

Los extremos superior e inferior de las probetas son descartables después del procesado por ECAP, ya que son zonas que no logran deformaciones apreciables en el material, por lo tanto, el punto de análisis debe ser la parte central de las probetas ECAP.

\section{Contribución de los autores}

La contribución al trabajo por parte de los autores se realizó de la siguiente manera: Luis Carlos Flórez y Cristian Pedraza plantearon el modelo de la simulación en ANSYS y Oscar Fabián Higuera analizó el efecto de la fricción y geometría de la matriz ECAP en el comportamiento de la aleación de aluminio 6061-T6.

\section{Referencias}

[1] H. Gleiter, "Nanocrystalline materials," Progress Materials Science, vol. 33, pp. 223-315,1989.

[2] Y. T. Zhu, T. C. Lowe, and T. G. Langdon, "Performance and applications of nanostructured materials produced by severe plastic deformation," Scripta Materialia, vol. 51 (8), pp. 825-830, Oct. 2004. DOI: https://doi.org/10.1016/i.scriptamat.2004.05.006.

[3] R. Z. Valiev, and T. G. Langdon, "Principles of equal-channel angular pressing as a processing tool for grain refinement," Progress in Materials Science, vol. 51 (7), pp.881-981, Sep. 2006. DOI: https://doi.org/10.1016/j.pmatsci.2006.02.003.

[4] V. M. Segal, "Materials processing by simple shear," Materials Science and Engineering A, vol. A197, pp.157-164, Jul. 1995. DOI: https://doi.org/10.1016/0921-5093(95)09705-8.

[5] R. Z. Valiev, N. A. Krasilnikov, and N. K. Tsenev, "Plastic deformation of alloys with submicron-grained structure," Materials Science and Engineering A, vol. 137, pp. 35-40. May. 1991. DOI: https://doi.org/10.1016/0921-5093(91)90316-F.

[6] R. Z. Valiev, "Structure and mechanical properties of ultrafine-grained metals," Materials Science and Engineering A, vol. 234-236, pp.59-66, Aug. 1997. DOI: https://doi.org/10.1016/S0921-5093(97)00183$\underline{4}$.

[7] Y. Saito, N. Tsuji, H. Utsunomiya, T. Sakai, and R. G. Hong, "Ultra-fine grained bulk aluminum produced by Accumulative Roll-Bonding (ARB) process," Scripta Materialia, vol. 39 (9), pp.1221-1227, Oct. 1998. DOI: https://doi.org/10.1016/S1359-6462(98)00302-9.

[8] Y. Saito, H. Utsunomiya, N. Tsuji, and T. Sakai, "Novel ultra-high straining process for bulk materialsdevelopment of the Accumulative Roll-Bonding (ARB) process," Acta Materialia, vol. 47 (2), pp. 579583, Jan. 1999. DOI: https://doi.org/10.1016/S1359-6454(98)00365-6.

[9] V. M. Segal, V. I. Reznikov, A. E. Drobyshevski, and V. I. Kopylov, "Plastic working of metals by simple shear," Russian Metallurgy, vol. 1, pp.99-105, 1981.

[10] Y. Iwahashi, J. Wang, Z. Horita, M. Nemoto, and T. G. Langdon, "Principle of equal-channel angular pressing for the processing of ultra-fine grained materials," Scripta Materialia, vol. 35 (2), pp.143-146, Jul. 1996. DOI: https://doi.org/10.1016/1359-6462(96)00107-8.

[11] ASM Handbook, Properties and selection: Nonferrous alloys and special-purpose materials. Edition Metals Handbook. ASM International Handbook Committee, 1990.

[12] J. R. Bowen, A. Gholinia, S. M. Roberts, and P. B. Prangnell, "Analysis of the billet deformation behaviour in equal channel angular extrusion," Materials Science and Engineering A, vol. 287 (1), pp. 87-99, Jul. 2000. DOI: https://doi.org/10.1016/S0921-5093(00)00834-0.

[13] A. Krishnaiah, K. Kumaran, and U. Chakkingal, "Finite element analysis of multi-pass equal channel angular extrusion/pressing process," Materials Science Forum, vol. 654-656, pp.1574-1577, Jun. 2010. DOI: https://doi.org/10.4028/www.scientific.net/MSF.654-656.1574. 